OPEN ACCESS

Edited by:

David Hughes,

Pennsylvania State University,

United States

Reviewed by:

Jutta M. Schneider,

University of Hamburg, Germany

Ximena J. Nelson,

University of Canterbury, New Zealand

*Correspondence:

Freddie-Jeanne Richard freddie.jeanne.richard@univ-poitiers.fr

Specialty section:

This article was submitted to Behavioral and Evolutionary Ecology,

a section of the journal

Frontiers in Ecology and Evolution

Received: 19 July 2017

Accepted: 07 November 2017

Published: 24 November 2017

Citation:

Richard F-J (2017) Symbiotic Bacteria Influence the Odor and Mating

Preference of Their Hosts.

Front. Ecol. Evol. 5:143.

doi: 10.3389/fevo.2017.00143

\section{Symbiotic Bacteria Influence the Odor and Mating Preference of Their Hosts}

\author{
Freddie-Jeanne Richard*
}

Team Ecologie Evolution Symbiose, Laboratoire Ecologie et Biologie des Interactions, UMR Centre National de la Recherche Scientifique 7267, Université de Poitiers, Poitiers, France

In many species, chemical communication is a determining factor in mate choice. Some species use the composition of cuticular compounds to discriminate between potential mates. Moreover, the presence of parasites can also influence mate choice and alter the odor of an individual. In the current study, we tested the effect of the endosymbiont Wolbachia, which is naturally present in the terrestrial isopod Armadillidium vulgare and can induce male feminization in the species. We compared male attraction to different types of females (Wolbachia-free, females naturally infected by Wolbachia, Wolbachia-injected females, and females injected with a bacteria-free suspension). Our behavioral assays revealed that males consistently preferred Wolbachia-free females and that this preference correlates to changes in cuticular compounds. Males also preferred naturally infected females over Wolbachia-injected females. The ability of males to discriminate between females could result in Wolbachia impacting its host phenotype, thus affecting the odor of the host. Female odor is the result of an honest signal that makes it possible for males to discriminate between Wolbachia-infected and non-infected females. The male preference for Wolbachia-free females is adaptive, and long-term studies have demonstrated that such choices confer improved fitness.

Keywords: chemical compounds, individual perception, feminization, individual odor, Armadillidium vulgare

\section{LAY SUMMARY}

A key aspect of sexual selection is the competition for mates. Individuals use specific traits to recognize and choose their partners. In gregarious species, individuals are in groups, which makes it easier to find a mate. We showed that males feminized into functional females due to infection by Wolbachia bacteria (i.e., male genotype and female phenotype) have a specific odor and are less attractive to males compared to females not infected with the bacteria.

\section{INTRODUCTION}

Many insects use volatile or contact pheromones (cues) located on the surface of individual cuticles to exchange information and communicate (Ginzel et al., 2003; Howard and Blomquist, 2005; Richard and Hunt, 2013). For example, chemical signals are part of sexual communication in a wide range of species and thus could be involved in the sexual selection mechanism of mate choice (Andersson, 1994; Johansson and Jones, 2007). Cuticular compounds are cues that communicate status, recognition (caste, nestmate, or mate), health, parasite load, and immune system 
(Fukaya et al., 2000; Ginzel et al., 2003; Richard et al., 2007, 2008, 2012; Ali and Tallamy, 2010; Liebig, 2010; Sharon et al., 2010; Richard and Hunt, 2013). More specifically, cuticular compounds can also be directly involved in mate recognition and affect female attractiveness to males (in Coleoptera: Cerambycidae, Ginzel et al., 2003 and Chrysomelidae, Ali and Tallamy, 2010). In honey bees, immune system activation via bacterial infection can also alter cuticular compound patterns used for nestmate recognition (Richard et al., 2008, 2012); additionally, fly microbiota (commensal bacteria) can have consequences on mating preferences of Drosophila (Sharon et al., 2010). The use of chemical cues to evaluate mates, specifically the health of a mate (bacterial infection), is addressed in the current study.

Mate choice and immunity are linked by the indirect fitness benefits of producing offspring with stronger immune systems and by the direct fitness impact of minimizing interactions or avoiding sick partners (Hamilton and Zuk, 1982; Loehle, 1997). Adaptive mate choice could also be a key factor in the evolutionary arms race between the host and its parasite in terms of survival and reproductive success. There is a conflict of interest for both hosts and parasites in regards to reproduction; the evolutionary strategies of the hosts consist of avoiding or slowing down the transmission of parasites, while the symbionts need to increase their own transmission (Combes, 2001). Infection can have negative impacts on the host phenotype, including ornamentation, coloration, calling, odors, and behavior (Beltran and Richard, 2014). Changes in the sexual trait phenotype due to parasites or endosymbiotic bacteria may then provide cues that indicate individual condition (Vergara et al., 2012) and could be considered as an honest signal of mate quality.

Numerous studies have highlighted the complexity of the symbiotic bacteria Wolbachia, in terms of its impact on its host's reproductive organs and somatic tissues (Miller and Schneider, 2012) and its accumulation in the nervous system (Strunov et al., 2013; Dittmer et al., 2014). Wolbachia are one of the most prevalent gram-negative bacteria in arthropods (Werren and Windsor, 2000; Hilgenboecker et al., 2008; Serbus et al., 2008; Cordaux et al., 2012). Wolbachia are obligate intracellular alpha-Proteobacteria that affect phenotypic traits in their hosts, extending from parasitism to mutualism (Riegler and O'Neill, 2007; Cook and McGraw, 2010). The coevolution of Wolbachia and their arthropod hosts may be driven by conflicts of interest, and the relationship may switch from a mutually beneficial one to an exploitative one depending on the environment (Herre et al., 1999; Wernegreen, 2004). The reproductive phenotypes obtained from Wolbachia that are associated with reproductive parasitism favor the fitness of infected females through cytoplasmic incompatibility (CI), which results in the embryonic mortality among offspring, sex ratio biases, which are weighted toward females in host progenies due to the killing of males (death of male progeny), parthenogenesis, or feminization, which converts genetic males into functional females (Miller and Schneider, 2012). The feminized males mimic female phenotypes at the morphological, reproductive and behavioral levels (Miller and Schneider, 2012). Wolbachia replicate inside the cells of their host, and they are predominantly transmitted through mother-offspring relationships (Bright and Bulgheresi, 2010).
As a consequence, by acting as sex-ratio distorters in favor of females, they also enhance their own transmission (Moreau and Rigaud, 2000; Cordaux et al., 2011). In general, Wolbachia are weakly virulent to their native hosts (Engelstädter and Hurst, 2009), probably as a result of the host/parasite coevolution of the vertical transmission process (Lipsitch et al., 1996; Engelstädter and Hurst, 2009), although the symbiotic bacteria may become pathogenic when bacterial levels get too high (Sachs and Simms, 2006; Le Clec'h et al., 2012). However, although Wolbachia affects the mating choice through CI of its host in some Drosophila (Moreau et al., 2001; Markov et al., 2009; Sharon et al., 2010), this is not always true for all Drosophila species (Jenkins et al., 1996; Sullivan and Jaenike, 2006).

In the terrestrial isopod Armadillidium vulgare, male embryos that inherit Wolbachia develop into functional females, and Wolbachia prevents the development of the androgenic gland. This gland secretes the androgenic hormone responsible for the differentiation of the male sexual phenotype. Wolbachia are thought to inhibit male gonad differentiation and convert genetic males into phenotypic females, leading to functional females. Infected females suffer fitness disadvantages and various costs, such as lower survival (Braquart-Varnier et al., 2008), lower learning and memory performance (Templé and Richard, 2015), and lower copulation investment (Moreau et al., 2001), which partially explains the low prevalence of feminizing Wolbachia in natural populations (Rigaud et al., 1999).

Despite the number of studies on the effects of Wolbachia on host fitness and on the prevalence and variability of their populations, there is a lack of detailed information on the role of feminizing bacteria on the mating preference of their hosts, and the proximate mechanisms underlying such choices are still unknown. The objectives of the current work are to test whether discrimination associated with sexual selection against Wolbachia infection exists by evaluating how attracted males are to Wolbachia-free and Wolbachia-infected females during the reproductive period and whether this individual discrimination can be effective at short distances without direct contact. Chemical signatures between individuals were compared to determine if chemical communication can be modified by Wolbachia-infection and to assess if the cuticular compound can be used as an indicator or an honest signal of Wolbachia infection status. Finally, we treated Wolbachia-free individuals with the cuticular extracts of control or Wolbachia-infected females and examined the interactions between males and coated individuals to determine if changes in the chemical profiles could indeed be responsible for the altered levels of attractiveness.

\section{MATERIALS AND METHODS}

Specimens of Armadillidium vulgare (Crustacea, Isopoda, Oniscidea; Latreille, 1804) were derived from individuals collected in Denmark (Helsingor) to provide both aposymbiotic females and females infected by a feminizing Wolbachia strain ( $w$ VulC) (Rigaud et al., 1991; Cordaux et al., 2004). A. vulgare males were considered to be uninfected by Wolbachia (i.e., Wolbachia-free). The specimens were kept in laboratory conditions $\left(20^{\circ} \mathrm{C}\right.$, natural photoperiod of France, $\left.46^{\circ} 40^{\prime} \mathrm{N}\right)$. 
Every spring, gravid females were isolated. Male and female offspring were separated into different boxes before reaching sexual maturity, allowing the breeding to be controlled. Individuals were kept in plastic boxes $(26 \times 13 \mathrm{~cm})$ on moistened compost and fed with slices of fresh carrots and lime leaves ad libitum.

The presence or absence of Wolbachia was investigated. The infection status of 10 randomly selected uninfected females and 10 randomly selected females naturally infected by Wolbachia was determined by DNA extraction and PCR assays of dissected gonads and nerve cords (Bouchon et al., 1998). Total genomic DNA was obtained by phenol extraction and ethanol precipitation (Kocher et al., 1989) from dissected gonads and nerve cords. Dissection and DNA extraction were performed under sterile conditions. For the PCR amplification, we used general 205-bp fragments of the wsp gene (primers 208F: $5^{\prime}$-TGG-TGC-AGC-ATT-TAC-TCC-AG-30 and 413R: 5' -TCGCTT-GAT-AAG-CAA-AAC-CA-30) to amplify a homologous $w s p$ fragment coding for a Wolbachia surface protein in all the diverse Wolbachia strains found in arthropods (Braig et al., 1998). PCR cycling conditions were $95^{\circ} \mathrm{C}$ for $2 \mathrm{~min} ; 35$ cycles at $95^{\circ} \mathrm{C}$ for $1 \mathrm{~min}, 55^{\circ} \mathrm{C}$ for $1 \mathrm{~min}, 72^{\circ} \mathrm{C}$ for $1 \mathrm{~min} ; 72^{\circ} \mathrm{C}$ for $5 \mathrm{~min}$; and then held at $4^{\circ} \mathrm{C}$. The infection status was then observed by ethidium bromide staining on agarose gel, and the presence of Wolbachia was signaled by a PCR product of approximately $600 \mathrm{bp}$.

Wolbachia infection status was confirmed by PCR in tested tissues from Wolbachia-infected females, and the absence of Wolbachia in Wolbachia-free females was also confirmed.

\section{Choice Chamber}

To test the attractiveness of Armadillidium vulgare conspecifics, we used a Y-shaped choice chamber built in a Petri dish $(9.5 \mathrm{~cm}$ diameter) that was covered with a new filter paper between each experiment. The tunnels were separated into two sections by a mesh. This mesh was covered with opaque paper with tiny holes so that the air could pass through it at a constant flow rate (as described in Beauché and Richard, 2013).

At the beginning of the experiment, the isopods were placed in the sections located at the 3 extremities of the tunnels (Figure 1): the two target subjects were placed in sections (IIa) and (IIb), respectively, and the tested subject was placed in section (I) and was then able to move into the experimental device. The positions of the target individuals were inverted between each experiment. Test subjects were placed into their respective sections $15 \mathrm{~min}$ before the start of the experiment by a non-observer person, and then the tested individual was carefully transferred to position (I). The behavioral record started when the test subject entered the neutral section (NS). The different sections were delimited with a dotted line on the transparent cover of the Petri dish. When an individual's body crossed into two sections, the location of the head was recorded. The time spent in the left (LS) and right sections (RS) was monitored for $10 \mathrm{~min}$ using the program EthoLog 2.2 (Ottoni, 2000).

\section{Artificial Infection with Wolbachia}

Aposymbiotic or Wolbachia-free A. vulgare (1-year-old virgin females) were infected with the Wolbachia strain $w$ VulC. For

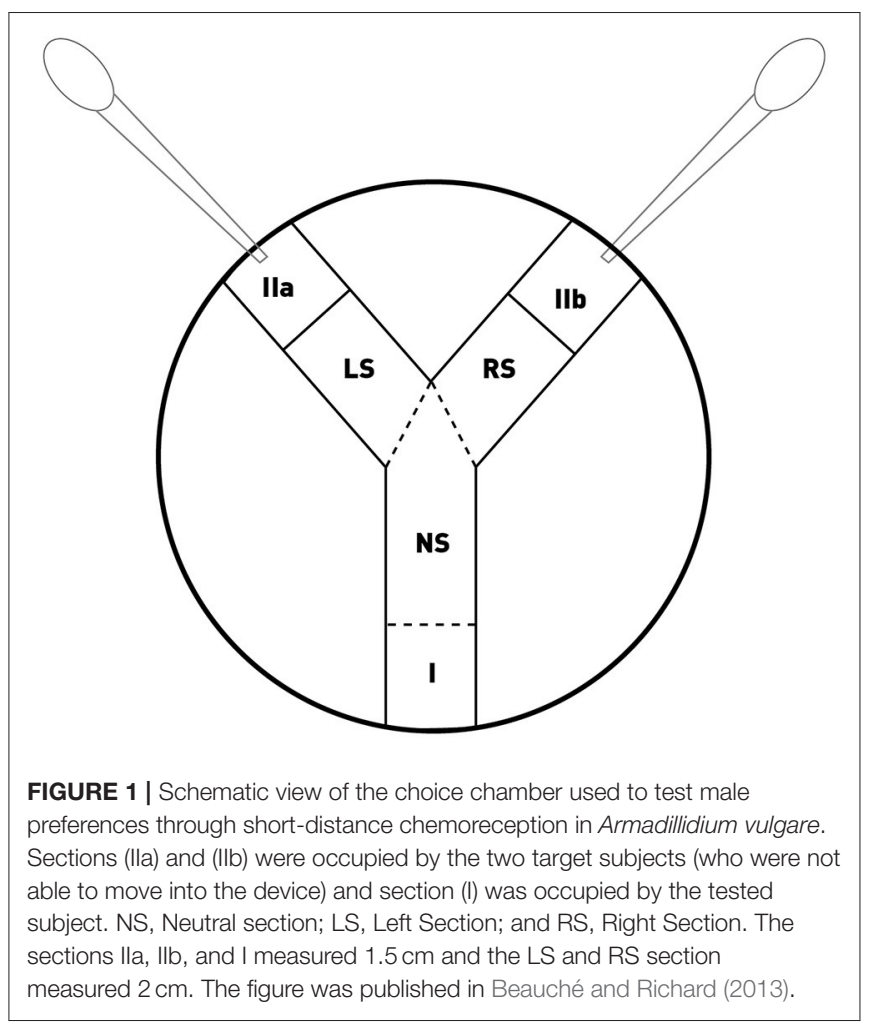

the injection, ovary suspensions were prepared using the ovaries of five A. vulgare symbiotically associated with $w \mathrm{VulC}$. The ovaries were collected and crushed into $1 \mathrm{ml}$ of Ringer solution. The resulting suspension was filtered through a $1.2-\mu \mathrm{m}$ pore membrane, and $1 \mu \mathrm{l}$ of each filtrate was injected through a small hole pierced in each individual's cuticle at the posterior part of the hemocoel using a thin glass needle (Rigaud and Juchault, 1995). In the control group, the females were injected with ovary suspensions prepared from ovaries of aposymbiotic females. This protocol was applied to inject three separate batches of individuals. Previous studies revealed that $w$ VulC injections in A. vulgare females had no effect on different life history traits (Le Clec'h et al., 2012). The animals were then used for behavioral assays 6 months post-injection, leaving enough time for Wolbachia to spread and be incorporated into the host tissue, as is the case in naturally infected individuals (Juchault and Mocquard, 1989).

\section{Experimental Groups}

Experiments were conducted during the spring, which is the mating period for these animals in nature, under the same temperature and humidity conditions in which the woodlice were raised and in nearly dark conditions ( $\sim 1$ lux). All the females used in our experiment were virgins, of the same age, similar in size and fed the same diet.

One-year old virgin males and females were used in our experiments, which occurred during the reproductive season (parturial molt). Females were sorted according to their physiological status (molting stage). The different molts and steps of molting are very complex and have been schematized in a 
recent publication (Beauché and Richard, 2013). The molting and reproductive cycles of females are closely linked, and complete ovarian maturation is only achieved during the preparturial intermolt; additionally, the molting cycle can be divided into five stages (Drach and Tchernigovtzeff, 1967). Here, females were in two different stages of the preparturial intermolt, during which females reach maximum activity regarding ovarian maturation, hormonal, physiological changes. They were tested either when in di-ecdysis or at the beginning of pre-ecdysis, before the appearance of white plates (C/D0 period, later called "pre-molt" and abbreviated as PM), or when white calcium plates appeared (in the middle of pro-ecdysis or in the D1 period, later called "early molt" and abbreviated as EM). In the choice test between Wolbachia-free females, males are more attracted to females in "early molt" compared to those in "pre-molt" (Beauché and Richard, 2013). Wolbachia-free males were all tested in "premolt" (C/D0).

The two experiments were completed in two consecutive years (2009 and 2010). All the samples were pooled, as no significant differences were obtained between combinations of individuals with the same condition.

In the first experiment, male attraction was measured between the two sections, one with the Wolbachia-free female and the other with the female naturally infected by Wolbachia; females were either both in pre-molt or both in early molt $(N=37$ and $N$ $=38$, respectively).

In the second experiment, male attraction was measured between the two sections, one with a target symbiotic (naturally infected) female and the other with a Wolbachia-injected female (injected 6 months prior to experiments), both in pre-molt; the second choice was between a control-injected female or a Wolbachia-injected female, both in pre-molt $(N=18$ and $N=23$, respectively).

\section{Effect of Natural Wolbachia-Infected Female Cuticular Extract on Male Attraction}

Male attraction was measured with two dead females that had been washed three times for $24 \mathrm{~h}$ in dichloromethane and coated with the cuticular extracts of either natural Wolbachia-infected or Wolbachia-free females. Different individuals (males and dead females) were used for each replicate $(N=24)$. Individuals were tested in a round Petri dish. The behavioral record started when the test subject started walking. The time spent near dead females coated with odors from other individuals was monitored for $10 \mathrm{~min}$ using the program EthoLog 2.2. The experiment was recorded and analyzed by different observers.

\section{Chemical Analysis}

Individuals (males, Wolbachia-free females and natural Wolbachia-infected females in pre-molt and early molt) were collected and frozen at $-20^{\circ} \mathrm{C}$ after the behavioral assays. The cuticular compounds were extracted by submerging each frozen individual into $1 \mathrm{ml}$ of dichloromethane with $0.2 \mu \mathrm{g}$ of pentadecane (Sigma, St. Louis, MO, U.S.A.) as an internal standard; samples were then held for $5 \mathrm{~min}$ under ultrasonic waves, gently agitated for $24 \mathrm{~h}$ and again held for 5 min under ultrasonic waves. The solution was then filtered and reduced to dryness with a gentle stream of N2, and the sample was re-suspended in $50 \mu \mathrm{l}$ of dichloromethane. The cuticular compounds were observed using gas chromatography. We analyzed $2 \mu \mathrm{l}$ of solution using a 7890 GC-FID (Agilent). A splitless injection was made into an HP-5 capillary column $(30 \mathrm{~m} \times 0.32 \mathrm{~mm} \times 1 \mu \mathrm{m})$ operated at $140^{\circ} \mathrm{C}$ for $2 \mathrm{~min}$, increased by $5^{\circ} \mathrm{C} / \mathrm{min}$ to $300^{\circ} \mathrm{C}$ and kept at this temperature for $15 \mathrm{~min}$. The injector and detector were kept at $300^{\circ} \mathrm{C}$. This protocol is a modified version of that used by Richard et al. (2004).

In total, between 5 and 8 individuals were tested for each category: males, Wolbachia-free females and natural Wolbachiainfected females in pre-molt and Wolbachia-free females and natural Wolbachia-infected females in early molt.

\section{Statistical Analysis}

The average choice for the arch containing the symbiotic female vs. the Wolbachia-free female in pre- and early-molt stages was statistically tested with sign tests, so only the time spent in the RS and LS sections was considered and compared using a non-parametric Wilcoxon test for dependent variables. The attractiveness of target individuals was calculated as the median time \pm semi-inter-quartile ranges (in seconds) the tested individuals spent in front of their section (i.e., in the right and/or left section).

To assess the similarity of odor profiles, a stepwise discriminant analysis was employed using Statistica 6.0. (StatSoft Inc.). Prior to analysis, each peak area was standardized according to Reyment (1989). Only peaks that were quantifiably reliable and reproducible were used for the statistical analysis; peaks that were consistently below $0.1 \%$ of the total quantity were omitted. Gas chromatography analysis of cuticular compounds was conducted as outlined in Richard et al. (2007).

\section{RESULTS}

\section{First Experiment}

Armadillidium vulgare males spent significantly more time in the section close to the uninfected females compared to the natural Wolbachia-infected females when they were in pre-molt (Wilcoxon: $N=37, T=89, p<0.0001$, Figure 2A) and when they were in early molt (Wilcoxon: $N=38, T=111$; $p<0.001$, Figure 2A). Only 17 males out of the 75 tested visited only one section: two males went only into the section with the Wolbachiainfected females, and the others only went into the section with Wolbachia-free females.

\section{Second Experiment}

Armadillidium vulgare males spent significantly more time in the section close to the control-injected females compared to the Wolbachia-injected females in pre-molt (Wilcoxon: $N=23 ; T=$ $57 ; p=0.013$, Figure 2B), and they spent more time close to the natural Wolbachia-infected females compared to the Wolbachiainjected females in pre-molt (Wilcoxon: $N=18$; $T=18$; $p=$ 

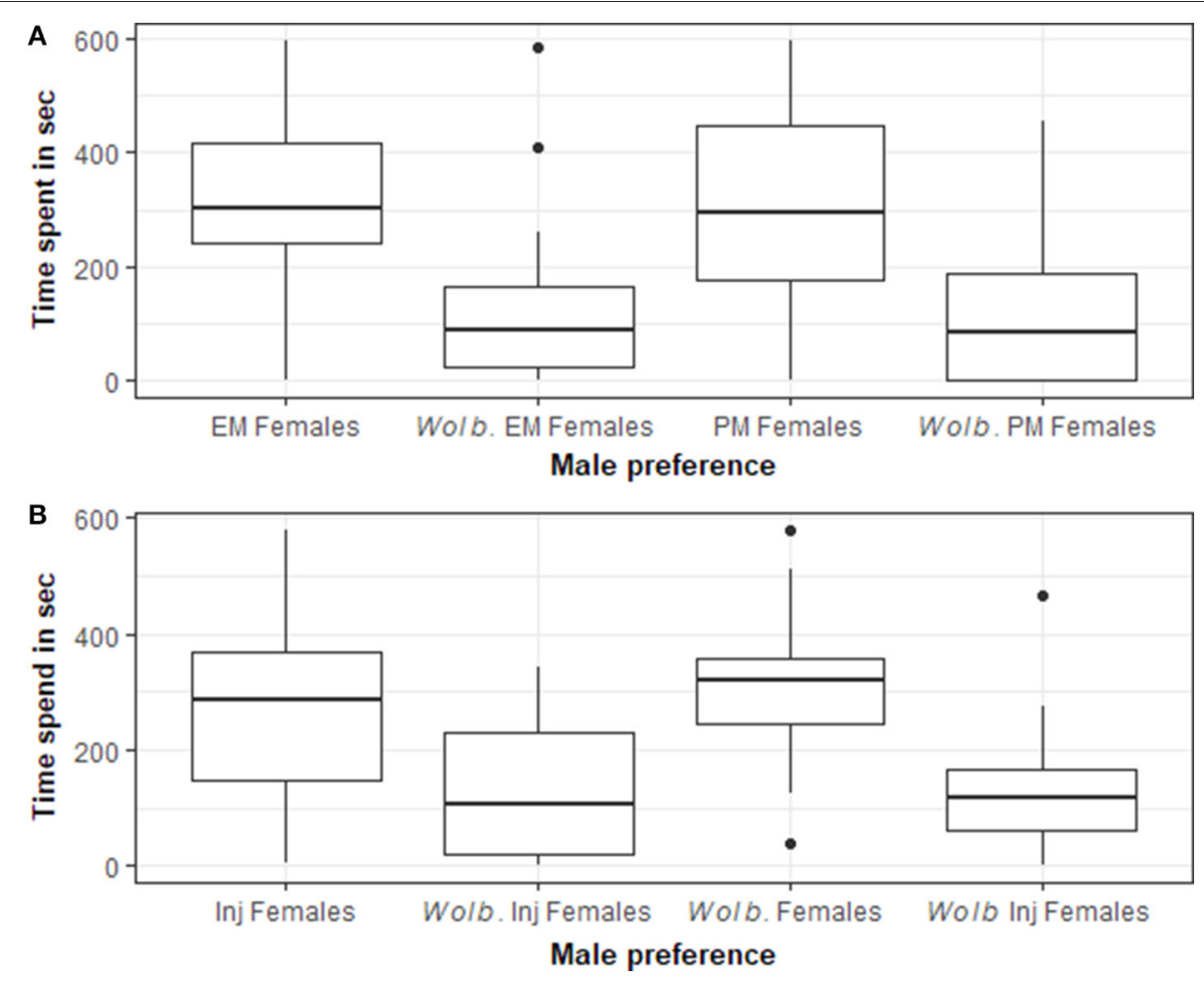

FIGURE 2 | Armadillidium vulgare males spent significantly more time in the section close to Wolbachia-free females compared to natural Wolbachia-infected females. (A) PM Females or EM Females: Wolbachia-free females in pre-molt (PM) or in early molt (EM); Wolb PM Females or Wolb EM Females: infected females in pre-molt or in early molt (in pre-molt Wilcoxon: $T=89 ; N=37 ; p<0.0001$ and in early molt Wilcoxon: $T=111 ; N=38 ; p<0.001$ ). (B) Armadillidium vulgare males spent significantly more time in the section close to control-injected females (Inj Females) compared to Wolbachia-injected females (Wolb Inj Females), both in pre-molt (Wilcoxon: $N=23 ; T=57 ; p=0.013$ ). Armadillidium vulgare males spent significantly more time in the section close to natural Wolbachia-infected females (Wolb Females) compared to Wolbachia-injected females (Wolb Inj Females), both in pre-molt (Wilcoxon: $N=18 ; T=18 ; p=0.0032)$.

0.0032, Figure 2B). Only 6 males out of the 41 tested visited only one section, and none of them visited the section with Wolbachia-injected females.

\section{Effect of Symbiotic Female Cuticular Extract on Male Attraction}

Armadillidium vulgare males spent significantly more time next to dead females coated with the cuticular extract of Wolbachiafree females than the cuticular extract of natural Wolbachiainfected females $(73 \% \pm 20$ and $13 \% \pm 28$ of their time, respectively; Wilcoxon: $N=24 ; T=65 ; p<0.008)$.

\section{Chemical Analysis}

Discriminant analysis was performed on the cuticular chemical compounds of males, Wolbachia-free females and natural Wolbachia-infected females in pre-molt and early-molt stages, based on their relative proportions. Chemical analysis of the cuticle revealed significant quantitative differences between males, natural Wolbachia-infected females and Wolbachia-free females $\left[F_{(64,115)}=2.46 ; p<0.0001\right.$; Figure 3]. Mahalanobis chemical distances were significantly different between each group ( $\mathrm{MD}>89 ; p<10-4)$.

\section{DISCUSSION}

Mate selection involves individuals choosing to mate with a specific individual over other equally accessible individuals. In many species throughout the animal kingdom, pre-mating processes to attract and select mates involve chemical signals. Attraction via air-borne info-chemicals enables individuals to perceive conspecifics and discriminate individual gender and molting stage in terrestrial isopods (Beauché and Richard, 2013). Our results clearly show effective chemical perception through volatile compounds at a distance of several centimeters. We demonstrated that in $A$. vulgare, males discriminate between Wolbachia-free and Wolbachia-infected females based on chemical perception. Females infected with Wolbachia have lower mating rates compared to Wolbachia-free females (Moreau et al., 2001). When males encounter both Wolbachiafree and Wolbachia-infected females, they make more mating attempts with Wolbachia-free females (Moreau et al., 2001). The correlation between the differences in the cuticular chemical profiles and the male preference for Wolbachia-free females may suggest that cuticular compounds are involved in mate recognition and female attractiveness in A. vulgare. Male mate choice could be the result of a specific signal that makes Wolbachia-free females more attractive at short distances. Sex 


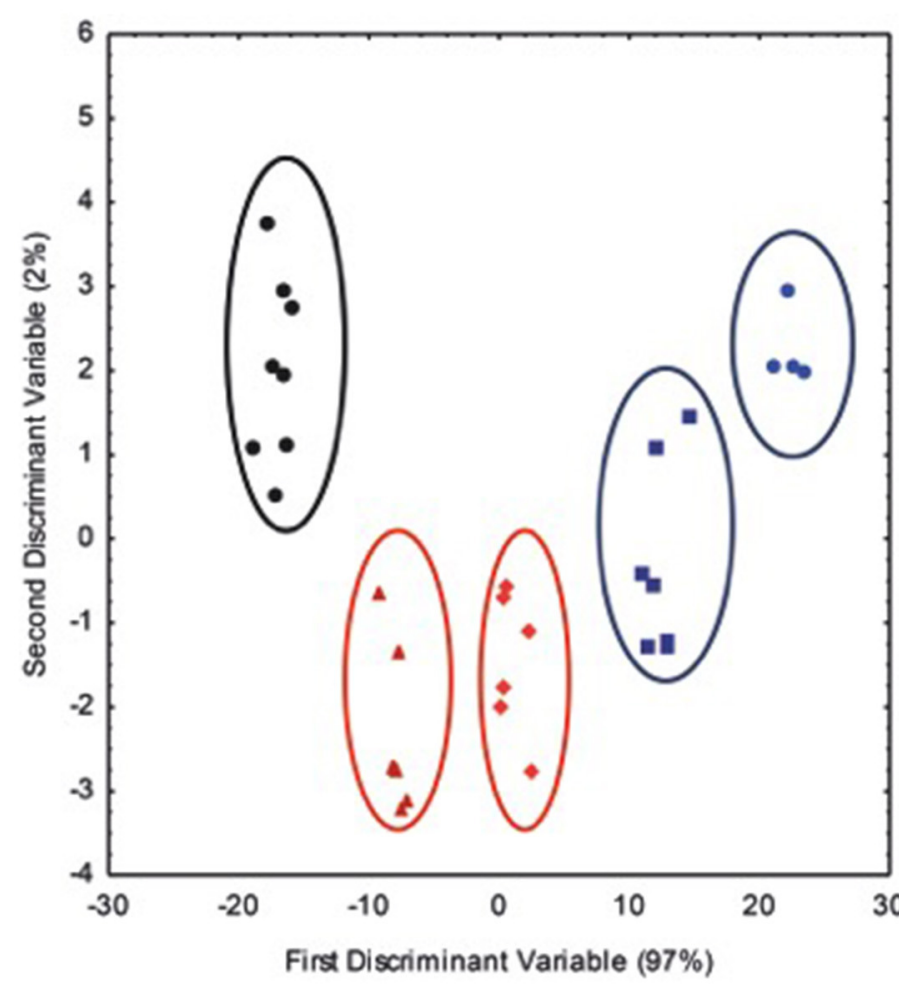

- Females PM
- Females EM
- Females Wolbachia PM
- Males

FIGURE 3 | Chemical profile analysis of the males, Wolbachia-free females, and symbiotic females. A discriminant analysis was performed on the cuticular chemical profiles of males, Wolbachia-free females in the pre-molt period (PM Female) and in the D1 Period (EM Female) and symbiotic females in the C/D0 period (Wolb PM Female) and in early-molt period (Wolb EM Female), based on the relative proportions of chemical compounds $\left[F_{(64,115)}=2.46 ; p<0.0001\right]$. Data were obtained from gas-chromatography analysis of cuticular extracts. Ellipses have been drawn to emphasize the categories but have no specific statistical meaning.

pheromones facilitate the initial attraction of sexual partners; here, they could be composed of a mixture of cuticular chemical compounds, with subtle but significant differences between Wolbachia-infected and non-infected individuals in our study model.

In the $A$. vulgare/Wolbachia host/parasite interaction, feminization converts genetic males into functional females, and as a consequence, accelerates the distribution of parasites through the host range. However, although males are less attracted to Wolbachia-infected females, they can still mate with them and invest less in the reproduction process compared to Wolbachia-free females (Moreau et al., 2001; Rigaud and Moreau, 2004). Lower mating rates of Wolbachia-infected females have been demonstrated in the butterfly Acraea encedon, in which the symbiont induces male-killing (Jiggins et al., 2000). Moreover, in the current study, males discriminate between Wolbachia-infected and Wolbachia-free females. Such discrimination could be the result of either incomplete feminization, meaning the Wolbachia-infected females have a chemical profile of combined male and female components, or it could be due to new compounds that are only synthesized by the infected host. Both genetics and environment (diet, physiology, gender, etc.) contribute to individual odors in many invertebrates (Richard and Hunt, 2013). Indeed, fly symbiotic bacteria, which are part of the normal fly microbiota, can influence mating preference by changing the levels of cuticular hydrocarbon sex pheromones, and antibiotic treatment abolished such preference (Sharon et al., 2010). In the case of feminization, Wolbachia transforms genotypic males (homogametic sex, ZZ) into phenotypic females ( $Z Z+$ Wo, also known as neo-females), so Wolbachia-infected females present male genotypes and female physiological characteristics, leading to imperfect feminization. Chemical profiles support this hypothesis, but the presence of other specific compounds due to the Wolbachia infection is still a possibility.

When selecting females based on cuticular chemical compounds, the males' non-preference for Wolbachia-infected females could be considered as a response to an honest signal of female parasite presence. There is evidence that Wolbachia induce various costs in terrestrial isopods, including reduced fertility and lower survival in Wolbachia-infected females (Rigaud and Juchault, 1998; Rigaud et al., 1999; Lachat et al., 2008). Females infected with Wolbachia differ markedly in fecundity, with a lower fitness due to males discriminating and allocating more time and more sperm to Wolbachia-free females in Armadillidium vulgare (Moreau et al., 2001). It is not known if the reduced fitness of Wolbachia-infected females is the direct consequence of the males' lower investment or the indirect effect of males selecting the most fecund females as mates, but after multiple matings, sperm depletion in males only affects fertility 
in the infected females (Rigaud and Moreau, 2004). In addition, in the same species, infected females are characterized by lower immunity compared to Wolbachia-free females (BraquartVarnier et al., 2008; Sicard et al., 2010). Behavioral differences between Wolbachia-free and Wolbachia-infected females were not qualitative, but the active discrimination between the two types of females confers an evolutionary advantage and better fitness when males choose Wolbachia-free females.

Naturally infected females are preferred to Wolbachia-injected females, suggesting that both types of females have different quantitative phenotypic traits compared to Wolbachia-free females. However, our behavioral results revealed that males can discriminate between these two types of females. A delay of 5 months between the bacterial injection and oocyte colonization was sufficient for vertical transfer (Juchault and Mocquard, 1989). Here, Wolbachia-injected females were tested 6 months postinjection. This time was largely sufficient to allow Wolbachia to colonize and become incorporated into the host tissues in the same way as naturally infected females. The phenotypic cues used for male mate choice, causing them to avoid the Wolbachiainjected individuals, suggest that males are more attracted to females that confer immunity against Wolbachia during their development (naturally infected females) than newly exposed females (Wolbachia-injected). Such a choice could potentially accrue indirect fitness benefits for the male by selecting naturally infected females. Moreover, even if we breed Wolbachia-injected females in the same condition as other females, the breeding success is lower, as most of them do not produce offspring (FJ Richard, personal communication). Males may also discriminate low fecundity but this hypothesis remains unknown.

Parasites and pathogens are a ubiquitous threat to organisms and may reduce host fitness by decreasing survival or reproductive success (Moreau et al., 2002). Our model provides an example of host/parasite interaction complexity. The impact of endosymbiont on host mate choice, demonstrated here, could then slow down or avoid parasite transmission. Infected individuals present a different phenotype that reduces the individual's attractiveness and supports male mating with lower

\section{REFERENCES}

Ali, J. G., and Tallamy, D. W. (2010). Female spotted cucumber beetles use own cuticular hydrocarbon signature to choose immunocompatible mates. Anim. Behav. 80, 9-12. doi: 10.1016/j.anbehav.2010.03.014

Andersson, M. (1994). Sexual Selection. Princeton, NJ: Princeton University Press.

Beauché, F., and Richard, F.-J. (2013). The best timing of mate search in Armadillidium vulgare (Isopoda, Oniscidea). PLOS ONE 8:e57737. doi: 10.1371/journal.pone.0057737

Beltran, S., and Richard, F. J. (2014). Impact of infection on mate choice. Anim. Behav. 90, 159-170. doi: 10.1016/j.anbehav.2014.01.026

Bouchon, D., Cordaux, R., and Greve, P. (2008). "Feminizing Wolbachia and the evolution of sex determination in isopods," in Insect Symbiosis, eds K. Bourtzis and T. Miller (Boca Raton, FL: Taylor and Francis Group LLC), 273-294.

Bouchon, D., Rigaud, T., and Juchault, P. (1998). Evidence for widespread Wolbachia infection in isopod crustaceans: molecular identification and host feminization. Proc. Biol. Sci. 265, 1081-1090. doi: 10.1098/rspb.1998.0402

Braig, H. R., Zhou, W., Dobson, S. L., and O'Neill, S. L. (1998). Cloning and characterization of a gene encoding the major surface protein of the bacterial endosymbiont Wolbachia pipientis. J. Bacteriol. 180, 2373-2378. investment. The trade-off for males, i.e., between low-investment mating with infected females vs. not mating, sustains the parasite in the population, and due to the biased sex-ratio, the transmission rate will increase with each generation (Bouchon et al., 2008). In addition to the various costs induced by Wolbachia, certain studies have revealed that these types of parasites can protect their hosts against other natural enemies and virulent horizontally transmitted parasites, which may have important implications in parasite co-evolution (Haine et al., 2005; Jones et al., 2010), with hosts becoming resistant to a variety of parasites (Kambris et al., 2009, 2010). The Armadillidium vulgare/Wolbachia model provides an illustration of the hypothesis put forward by Hamilton and Zuk (1982), which states that the evolutionary interactions between hosts/pathogens and mate choice affects both the direct and indirect fitness of the hosts.

\section{AUTHOR CONTRIBUTIONS}

F-JR: Conceived and designed the experiments, contribute to behavioral observations, analyzed the data and wrote the paper.

\section{FUNDING}

This work was supported by the State-Region Planning Contracts (CPER) and the European Regional Development fund (FEDER).

\section{ACKNOWLEDGMENTS}

I would like to thank Pauline Bonnenfant, Camille Houdelet, Bryce Williamson, and Mathieu Sicard for their contributions to the behavioral observations performed blind and Catherine Debenest, Maryline Raimond for their technical support. This manuscript was proofread for English quality by American Journal expert. This work was supported by the Centre National pour la Recherche Scientifique and the French ministère de l'Enseignement Supérieur et de la Recherche.

Braquart-Varnier, C., Lachat, M., Herbinière, J., Johnson, M., Caubet, Y., Bouchon, D., et al. (2008). Wolbachia mediate variation of host immunocompetence. PLoS ONE 3:e3286. doi: 10.1371/journal.pone.0003286

Bright, M., and Bulgheresi, S. (2010). A complex juorney: transmission of microbioal symbionts. Nat. Rev. Microbiol. 8, 218-230. doi: 10.1038/nrmicro2262PMID:20157340

Combes, C. (2001). Parasitism: the Ecology and Evolution of Intimate Interactions. Chicago, IL; London: Chicago Press.

Cook, P. E., and McGraw, E. A. (2010). Wolbachia pipientis: an expanding bag of tricks to explore for disease control. Trends Parasitol. 26, 373-375. doi: 10.1016/j.pt.2010.05.006

Cordaux, R., Bouchon, D., and Grève, P. (2011). The impact of endosymbionts on the evolution of host sex-determination mechanisms. Trends Genet. 27, 332-341. doi: 10.1016/j.tig.2011.05.002

Cordaux, R., Michel-Salzat, A., Frelon-Raimond, M., Rigaud, T., and Bouchon, D. (2004). Evidence for a new feminizing Wolbachia strain in the isopod Armadillidium vulgare: evolutionary implications. Heredity 93, 78-84. doi: $10.1038 /$ sj.hdy. 6800482

Cordaux, R., Pichon, S., Hatira, H. B. A., Doublet, V., Grève, P., Marcadé I., et al. (2012). Widespread Wolbachia infection in terrestrial isopods 
and other crustaceans. Zookeys 176, 123-131. doi: 10.3897/zookeys.17 6.2284

Dittmer, J., Beltran-Bech, S., Lesobre, J., Raimond, M., Johnson, M., and Bouchon, D. (2014). Host tissues as microhabitats for Wolbachia and quantitative insights into the bacterial community in terrestrial isopods. Mol. Ecol. 23, 2619-2635. doi: $10.1111 / \mathrm{mec} .12760$

Drach, P., and Tchernigovtzeff, C. (1967). Sur lam ethode de détermination des stages d'intermue et son application générale aux Crustacés. Vie et milieux 18559, 595-609.

Engelstädter, J., and Hurst, G. D. D. (2009). The ecology and evolution of microbes that manipulate host reproduction. Annu. Rev. Ecol. Evol. Syst. 40, 127-149. doi: 10.1146/annurev.ecolsys.110308.120206

Fukaya, M., Akino, T., Yasuda, T., Wakamura, S., Satoda, S., and Senda, S. (2000). Hydrocarbon components in contact sex pheromone of the whitespotted longicorn beetle, Anoplophora malasiaca (Thomson) (Coleoptera: Cerambycidae), and pheromonal activity of synthetic hydrocarbons. Entomol. Sci. 3, 211-218. Available online at: http://ci.nii.ac.jp/naid/110003374659/en/

Ginzel, M. D., Blomquist, G. J., and Millar, J. G. (2003). Role of contact pheromones in mate recognition in Xylotrechus colonus. J. Chem. Ecol. 29, 533-545. doi: 10.1023/A:1022894419521

Haine, E. R., Boucansaud, K., and Rigaud, T. (2005). Conflict between parasites with different transmission strategies infecting an amphipod host. Proc. R. Soc. B 272, 2505-2510. doi: 10.1098/rspb.2005.3244

Hamilton, W., and Zuk, M. (1982). Heritable true fitness and bright birds: a role for parasites? Science 218, 384-387. doi: 10.1126/science.7123238

Herre, E. A., Knowlton, N., Mueller, U. G., and Rehner, S. A. (1999). The evolution of mutualisms: exploring the paths between conflict and cooperation. Trends Ecol. Evol. 14, 49-53. doi: 10.1016/S0169-5347(98)01529-8

Hilgenboecker, K., Hammerstein, P., Schlattmann, P., Telschow, A., and Werren, J. H. (2008). How many species are infected with Wolbachia? A statistical analysis of current data. FEMS Microbiol. Lett. 281, 215-220. doi: 10.1111/j.1574-6968.2008.01110.x

Howard, R. W., and Blomquist, G. J. (2005). Ecological, behavioral, and biochemical aspects of insect hydrocarbons. Ann. Rev. Entomol. 50, 371-393. doi: 10.1146/annurev.ento.50.071803.130359

Jenkins, T. M., Babcock, C. S., Geiser, D. M., and Anderson, W. W. (1996). Cytoplasmic incompatibility and mating preference in Colombian Drosophila pseudoobscura. Genetics 142, 189-194.

Jiggins, F. M., Hurst, G. D. D., and Majerus, M. E. N. (2000). Sex ratio distorting Wolbachia cause sex-role reversal in its butterfly bosts. Proc. R. Soc. B 267, 69-73. doi: 10.1098/rspb.2000.0968

Johansson, B. G., and Jones, T. M. (2007). The role of chemical communication in mate choice. Biol. Rev. Camb. Philos. Soc. 82, 265-289. doi: 10.1111/j.1469-185X.2007.00009.x

Jones, E. O., White, A., and Boots, M. (2010). The evolutionary implications of conflict between parasites with different transmission modes. Evolution 64, 2408-2416. doi: 10.1111/j.1558-5646.2010.00992.x

Juchault, P., and Mocquard, J. P. (1989)., Effet de l'inoculation d'une bactérie endocellulaire féminisante sur la croissance et la reproduction des femelles du crustacé Oniscoide Armadillidium vulgare (Latr.). Crustaceana 56, 83-92. doi: 10.1163/156854089X00824.

Kambris, Z., Blagborough, A. M., Pinto, S. B., Blagrove, M. S., Godfray, H. C., Sinden, R. E., et al. (2010). Wolbachia stimulates immune gene expression and inhibits plasmodium development in Anopheles gambiae. PLoS Pathog. 6:e1001143. doi: 10.1371/journal.ppat.1001143

Kambris, Z., Cook, P. E., Phuc, H. K., and Sinkins, S. P. (2009). Immune activation by life-shortening Wolbachia and reduced filarial competence in mosquitoes. Science 326, 134-136. doi: 10.1126/science.1177531

Kocher, T. D., Thomas, W. K., Meyer, A., Edwar, S. V., Pääbo, S., Villablanca, F. X., et al. (1989). Dynamics of mitochondrial DNA evolution in animals: amplification and sequencing with conserved primers. Proc. Natl. Acad. Sci. U.S.A. 86, 6196-6200. doi: 10.1073/pnas.86.16.6196

Lachat, M., Caubet, Y., and Bouchon, D. (2008). "Does Wolbachia influence survival in starved Armadillidium vulgare?" in Procceedings of International Symposium of Terrestrial Isopod ISTIB 07, eds M. Zimmer, F. CharfiCheikhrouha, and S. Taiti (Tunis: Verlag), 125-130.

Le Clec'h, W., Braquart-Varnier, C., Raimond, M., Ferdy, J.-B., Bouchon, D., and Sicard, M. (2012). High virulence of Wolbachia after host switching: when autophagy hurts. PLoS Pathog. 8:e1002844. doi: 10.1371/journal.ppat.1002844
Liebig, J. (2010). "Hydrocarbon profiles indicate fertility and dominance status in ant, bee and wasp colonies," in Insect Hydrocarbons: Biology, Biochemistry and Chemical Ecology, eds G. J. Blomquist and A. G. Bagnères (New York, NY: Cambridge University Press), 254-281.

Lipsitch, M., Siller, S., and Nowak, M. A. (1996). The evolution of virulence in pathogens with vertical and horizontal transmission. Evolution 50, 1729-1741. doi: 10.1111/j.1558-5646.1996.tb03560.x

Loehle, C. (1997). The pathogen transmission avoidance theory of sexual selection. Ecol. Model. 103, 231-250. doi: 10.1016/S0304-3800(97)00106-3

Markov, A. V., Lazebny, O. E., Goryacheva, I. I., Antipin, M. I., and Kulikov, A. M. (2009). Symbiotic bacteria affect mating choice in Drosophila melanogaster. Anim. Behav. 77, 1011-1017. doi: 10.1016/j.anbehav.2009.01.011

Miller, J. M., and Schneider, D. (2012). "Endosymbiotic microbes as adaptive manipulators or arthropod behavior and natural driving sources of host speciation," in Host Manipulation by Parasites, eds D. P. Hughes, J. Brodeur, and F. Thomas (Oxford, UK: Oxford University Press), 119-137.

Moreau, J., Bertin, A., Caubet, Y., and Rigaud, T. (2001). Sexual selection in an isopod with Wolbachia- induced sex reversal: males prefer real females. J. Evol. Biol. 14, 388-394. doi: 10.1046/j.1420-9101.2001.00292.x

Moreau, J., and Rigaud, T. (2000). Operational sex ratio in terrestrial isopods: interaction between potential rate of reproduction and Wolbachia-induced sex ratio distortion. Oikos 91, 477-484. doi: 10.1034/j.1600-0706.2000.910308.x

Moreau, J., Seguin, S., Caubet, Y., and Rigaud, T. (2002). Female remating and sperm competition patterns in a terrestrial crustacean. Anim. Behav. 64, 569-577. doi: 10.1006/anbe.2002.4000

Ottoni, E. B. (2000). EthoLog 2.2: a tool for the transcription and timing of behaviora observation session. Behav. Res. Meth. 32, 446-449. doi: 10.3758/BF03200814

Reyment, R. A. (1989). Compositional data analysis. Terra Rev. 1, 29-34. doi: 10.1111/j.1365-3121.1989.tb00322.x

Richard, F.-J., Aubert, A., and Grozinger, C. M. (2008). Modulation of social interactions by immune stimulation in honey bee workers, Apis mellifera. BMC Biol. 6:50. doi: 10.1186/1741-7007-6-50

Richard, F.-J., Hefetz, A., Christides, J. P., and Errard, C. (2004). Food influence on colonial recognition and chemical signature between nestmates in the fungus-growing ant Acromyrmex subterraneus. Chemoecology 14, 9-16. doi: 10.1007/s00049-003-0251-3

Richard, F.-J., Holly, H., and Grozinger, C. M. (2012). Effects of immunostimulation on social behavior, chemical communication and genome-wide gene expression in honey bee workers (Apis mellifera). BMC Genomics 13:558. doi: 10.1186/1471-2164-13-558

Richard, F.-J., and Hunt, J. H. (2013). Intracolony chemical communication in social insects. Insect. Soc. 60, 275-291. doi: 10.1007/s00040-013-0306-6

Richard, F.-J., Poulsen, M., Drijfhout, F., Jones, G. R., and Boomsma, J. J. (2007). Specificity in chemical profiles of workers, brood and mutualistic fungi in atta, acromyrmex, and sericomyrmex fungus-growing ants. J. Chem. Ecol. 33, 2281-2292. doi: 10.1007/s10886-007-9385-Z

Riegler, M., and O'Neill, S. L. (2007). Evolutionary dynamics of insect symbiont association. Trends Ecol. Evol. 22, 625-627. doi: 10.1016/j.tree.2007.08.013

Rigaud, T., and Juchault, P. (1995). Success and failure of horizontal transfers of feminizing Wolbachia endosymbionts in woodlice. J. Evol. Biol. 8, 249-255. doi: 10.1046/j.1420-9101.1995.8020249.x

Rigaud, T., and Juchault, P. (1998). Sterile intersexuality in an isopod induced by the interaction between a bacterium (Wolbachia) and the environment. Can. J. Zool. 76, 493-499. doi: 10.1139/z97-216

Rigaud, T., Moreau, J., and Juchault, P. (1999). Wolbachia infection in the terrestrial isopod Oniscus asellus: sex ratio distortion and effect on fecundity. Heredity 83, 469-475. doi: 10.1038/sj.hdy.6885990

Rigaud, T., and Moreau, M. (2004). A cost of Wolbachia-induced sex reversal and female-biased sex ratios: decrease in female fertility after sperm depletion in a terrestrial isopod. Proc. Biol. Sci. 271, 1941-1946. doi: 10.1098/rspb.2004.2804

Rigaud, T., Souty Grosset, C., Raimond, R., Mocquard, J. P., and Juchault, P. (1991). Feminizing endocytobiosis in the terrestrial crustacean Armadillidium vulgare Latr. (Isopoda): recent acquisitions. Endocytobiosis Cell Res. 7, 259-273.

Sachs, J. L., and Simms, E. L. (2006). Pathways to mutualism breakdown. Trends Ecol. Evol. 21, 585-592. doi: 10.1016/j.tree.2006.06.018

Serbus, L. R., Casper-Lindley, C., Landmann, F., and Sullivan, W. (2008). The genetics and cell biology of Wolbachia-host interactions. Annu. Rev. Genet. 42, 683-707. doi: 10.1146/annurev.genet.41.110306.130354 
Sharon, G., Segal, D., Ringo, J. M., Hefetz, A., Zilber-Rosenberg, I., and Rosenberg, E. (2010). Commensal bacteria play a role in mating preference of Drosophila melanogaster. Proc. Natl. Acad. Sci. U.S.A. 107, 20051-20056. doi: 10.1073/pnas.1009906107

Sicard, M., Chevalier, F., De Vlechouver, M., Bouchon, D., Grève, P., and Bouchon, D. (2010). Variation of immune parameters in terrestrial isopods: a matter of gender, aging and Wolbachia. Naturwissenschaften 97, 819-826. doi: 10.1007/s00114-010-0699-2

Strunov, A., Kiseleva, E., and Gottlieb, Y. (2013). Spatial and temporal distribution of pathogenic Wolbachia strain wMelPop in Drosophila melanogaster central nervous sytem under different temperature conditions. J. Inv. Pathol. 114, 22-30. doi: 10.1016/j.jip.2013.05.001

Sullivan, J., and Jaenike, J. (2006). Male-killing Wolbachia and male mate choice: a test with Drosophila innubila. Evol. Ecol. Res. 8, 91-102.

Templé N., and Richard, F.-J. (2015). Intra-cellular bacterial infections affect learning and memory capacities of an invertebrate. Front. Zool. 12:36. doi: 10.1186/s12983-015-0129-6

Vergara, P., Mougeot, F., Martinez-Padilla, J., Leckie, F., and Redpath, S. M. (2012). THe condition dependence of a secondary sexual trait is stronger under high parasite infection level. Behav. Ecol. 23, 502-511. doi: 10.1093/beheco/ arr216

Wernegreen, J. J. (2004). Endosymbiosis: lessons in conflict resolution. PLoS Biol. 2:e68. doi: 10.1371/journal.pbio.0020068

Werren, J. H., and Windsor, D. M. (2000). Wolbachia infection frequencies in insects: evidence of a global equilibrium? Proc. Biol. Sci. 267, 1277-1285. doi: $10.1098 / \mathrm{rspb} .2000 .1139$

Conflict of Interest Statement: The author declares that the research was conducted in the absence of any commercial or financial relationships that could be construed as a potential conflict of interest.

Copyright (C) 2017 Richard. This is an open-access article distributed under the terms of the Creative Commons Attribution License (CC BY). The use, distribution or reproduction in other forums is permitted, provided the original author(s) or licensor are credited and that the original publication in this journal is cited, in accordance with accepted academic practice. No use, distribution or reproduction is permitted which does not comply with these terms. 